\title{
Neuroendocrine regulatory role of Merkel cells in the pathogenesis of psoriasis
}

\author{
Taihua Liu“*, Defang Liu ${ }^{2}$, Xiaoxiang Wang ${ }^{3}$, Jun Wang1, Xiaojun Wang1 and Zhongkui Zhang ${ }^{1}$ \\ ${ }^{1}$ Department of Dermatology, General Hospital of Chengdu Military Region, Chengdu, 610083 China. \\ ${ }^{2}$ Department of Traditional Chinese Medicine, General Hospital of Chengdu Military Region, Chengdu, 610083 China. \\ ${ }^{3}$ Department of Presbyatrics, General Hospital of Chengdu Military Region, Chengdu, 610083 China.
}

Accepted 15 August, 2011

\begin{abstract}
It has been confirmed that neuroendocrine regulation involves in the pathogenesis of psoriasis, but the association between neuroendocrine and skin immune is still unknown. The present study aimed to explore the relationship of Merkel cells in the skin lesions and the pathogenesis of psoriasis. The ultrastructure of Merkel cells was observed under an electron microscope and immunohistochemistry was performed to detect the expressions of CK20, CK19, CK18, S100A7, SP and CD13 in the skin lesions of psoriasis patients and normal controls. Our results showed the neuroendocrine granules in the Merkel cells increased significantly at the progressive stage of psoriasis; the expressions of CK20, CK19 and CK18 in the skin lesions were markedly higher than those in normal controls, the CK20 expression at the progressive stage was significantly higher than that the initial stage and recovery stage; the expressions of S100A7, SP and CD13 in the skin lesions at the progressive stage were higher than those in the initial stage and recovery stage and positively related to the CK20 expression. Merkel cells may play an important neuroendocrine regulatory role in the pathogenesis of psoriasis.
\end{abstract}

Key words: Merkel cell, psoriasis neuroendocrine, pathogenesis.

\section{INTRODUCTION}

Shepherd proposed that immune function without nervous regulation may be sufficient for local responsiveness to subsequent challenge (Shepherd et al., 2005) and the nervous regulation played an important role in the homeostasis of immune function including skin immune. Since Misery put forward the concept of neuroimmuno-cutaneous system (NICS) in 1996 (Misery, 1996) and then, O'Sullivan (1998) concept of neruo-immunocutaneous-endocrine network (NICE) in 1998, increasing attention has been paid to the nervous regulation of skin immune which is now a focus in the studies of skin immune. The nervous system (especially the peripheral nerves) can regulate skin immune by secreting neuropeptides or neurotransmitters (Roosterman et al., 2006). Under or over regulation of immune system by nervous system may lead to immune dysfunction causing diseases. Research has demonstrated that the serum

\footnotetext{
${ }^{*}$ Corresponding author. E-mail: liu781234@sina.com.
}

levels of neuropeptides in the psoriasis patients including substances $\mathrm{P}$ (SP), vasoactive intestinal peptide (VIP) and calcitonin gene-related peptide (CGRP) are markedly higher than those in healthy controls (Reich et al., 2007), the nerve fibers in the skin lesions of patients with psoriasis vulgaris are dramatically longer than those in healthy subjects (Wang et al., 2004) and the nerve growth factor (NGF) expression in the skin lesions is significantly increased when compared with that in controls (Ye et al., 2005). For a long time, it was believed that Merkel cells were the skin receptive cells of mechanical stimulation signal.

Merkel cells and nerve endings formed a special anatomical connection which could deliver information on changes in peripheral and central command. In addition, Merkel cells expressed kinds of neuropeptides and neuropeptide receptors such as SP, VIP and CGRP through which to play an important role on the regulation of the skin immune system. Merkel cells may be the connection point of the anatomy and function between the neuroendocrine system and the immune system. 
These findings suggest neuroendocrine plays a critical role in the pathogenesis of psoriasis which are also supported by some recent studies (Karanikas et al., $2009,2007)$. Therefore, it is imperative to find the specific organs/cells that sense and transmit the information from susceptible factors, the way these organs/cells transmit the information to the advanced central nervous system (CNS), and the exact organs/cells receiving the commands from the advanced CNS and finally activating $T$ cells. Seen from the anatomy and neuroendocrinology, Merkel cells may have the potential to serve as perceiving the information of psoriasis etiology.

\section{MATERIALS AND METHODS}

\section{General information}

A total of 19 patients with psoriasis were randomly selected. These patients had no other diseases and had cutaneous lesions at different stages. There were 12 males and 7 females with a mean age of $34.28 \pm 12.17$ years (range: $19 \sim 58$ years). The mean course of disease was $6.21 \pm 6.77$ years (range: 2 months $\sim 30$ years) and the mean psoriasis severity score was $21.72 \pm 13.02$ (range: $5 \sim 53$ ). The study was approved by the ethics committee and obtained patient's consent.

\section{Sample collection}

Under the permission of the patients, 6 full-thickness affected skins were collected from each patient: 2 normal skins surrounding the affected skins (at the initial stage), 2 skin lesions (at the progressive stage) and 2 from the recovered lesions (taken to be at the recovery stage). One sample in each group was used for electron microscopy and the remaining samples applied for immunohistochemistry for CK20, CK19, CK18, S100A7, SP and CD13. In addition, normal skins were collected from 19 traumatic patients without psoriasis during the debridement. There were 15 males and 4 females with a mean age of $35.4 \pm 10.05$ years (range: $19 \sim 56$ years).

\section{Electron microscopy}

Electron microscopy (JEM.1010. 20, JEOL) was performed. Merkel cells and neuroendocrine granules in the cytoplasm were counted and the size of nucleus was measured. Twenty fields were randomly selected under light microscope in each sample for measuring optical density, calculating the average optical density of each sample, collecting and calculating the average optical density value.

\section{Reagents}

The primary antibodies against CK20, CK19, CK18, S100A7, SP and $C D 13$, the biotin labeled second antibody and the DAB chromogenic reagent were purchased from Beijing Zhongshan Golden Bridge Biology Technology Co. Ltd and used in the present study.

\section{Immunohistochemical staining}

Seven consecutive sections $(5 \mu \mathrm{m})$ were obtained from each sample. The glass slides were previously coated with 1:10 polylysine. Immunohistochemistry for CK20, CK19, CK18, S100A7, $\mathrm{SP}$ and CD13 was performed with 6 sections of each sample. After deparaffinization, antigen retrieval was carried out in $0.01 \mathrm{~mol} / \mathrm{L}$ citrate solution in a microwave oven (grade III) for $5 \mathrm{~min}$ and sections were allowed to cool to room temperature. Then, these sections were washed in TBS thrice $(3 \times 5 \mathrm{~min})$ and treated with $0.3 \% \mathrm{H}_{2} \mathrm{O}_{2}$ at room temperature for $30 \mathrm{~min}$. After washing with TBS thrice $(3 \times 5 \mathrm{~min})$, sections were incubated with $0.1 \%$ trito-X at $37^{\circ} \mathrm{C}$ for $30 \mathrm{~min}$ followed by washing with TBS thrice $(3 \times 5 \mathrm{~min})$. Subsequently, these sections were treated with normal goat serum $(1: 75)$ in humidified air at $37^{\circ} \mathrm{C}$ for $30 \mathrm{~min}$, primary antibodies (1:100) in humidified air at $4^{\circ} \mathrm{C}$ for $72 \mathrm{~h}$, washed with TBS thrice (3 $\times 5 \mathrm{~min})$ and then treated with secondary antibody $(1: 100)$ in humidified air at $37^{\circ} \mathrm{C}$ for $1 \mathrm{~h}$. After washing with TBS thrice $(3 \times 5$ min), $A B C$ complex was added followed by incubation in humidified air at $37^{\circ} \mathrm{C}$ for $1 \mathrm{~h}$ and then washing with TBS thrice $(3 \times 5 \mathrm{~min})$. Development was performed with DAB for $5 \mathrm{~min}$ and reaction was terminated by addition of distilled water.

After conventional dehydration and transparentization, the sections were mounted with neural gum. In negative controls, primary antibody was replaced with TBS and the remaining procedures were the same to those aforementioned.

\section{Image analysis}

Image-Pro plus (6.0.0.260) of Media Cybemetics, Inc. was used to detect the average optical density (OD) of the lesions with positive staining particles.

\section{Statistical analysis}

Statistical analysis was performed with SAS9.1 and MEANS applied to calculate the means and standard deviation $(X \pm S)$; $t$ test was applied for the comparisons of means between two groups. A value of $\mathrm{P}<0.05$ was considered statistically significant.

\section{RESULTS}

\section{Changes in the ultrastructure of Merkel cells}

Merkel cells localized in the basal layer of the epidermis were closely connected to the nerve endings with clear cytoplasm and irregular nucleus and were distributed between epidermal cells with digitations. Merkel cells had the features of epidermal cells while, under an electron microscope, neuroendocrine granules of $50 \sim 100 \mathrm{~nm}$ were seen. The nuclei of Merkel cell in the lesioned skins at the progressive stage had no obvious changes when compared with those at the initial and recovery stages, but, the neuroendocrine granules in the cytoplasm of Merkel cells in the lesioned skins at the progressive stage increased significantly when compared with those in cells at the initial and recovery stages (Figure 1). Generally, the old lesioned skin of psoriasis patients often disappeared with new lesions presented at the same time. Surrounding the lesions was normal skin that was visible which may be in fact the formation of early lesions, while the normal skin away from the lesions may be the truly normal skin. Therefore, skin close to the lesions, 

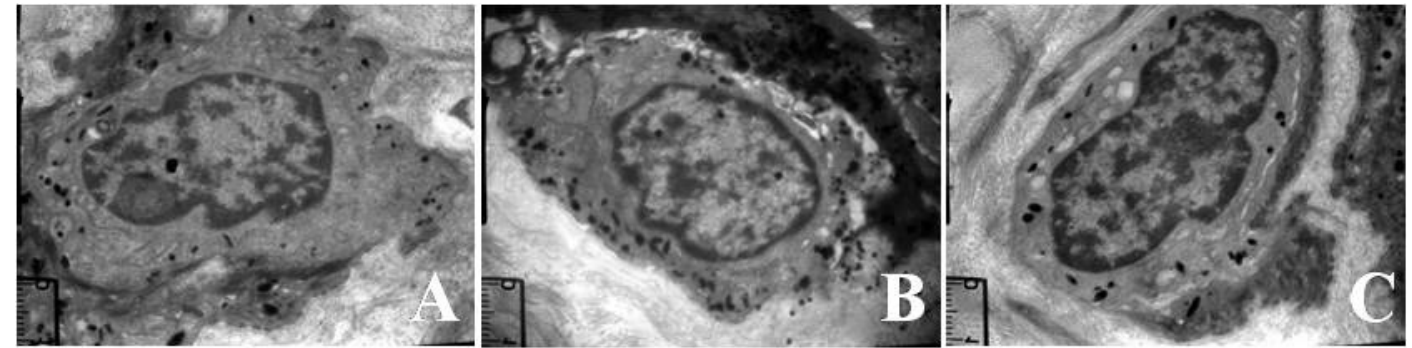

Figure 1. Ultrastructural features of Merkel cells (10000x). A: at initial stage; B: at progressive stage; C: at recovery stage (arrow represents neuroendocrine granules).

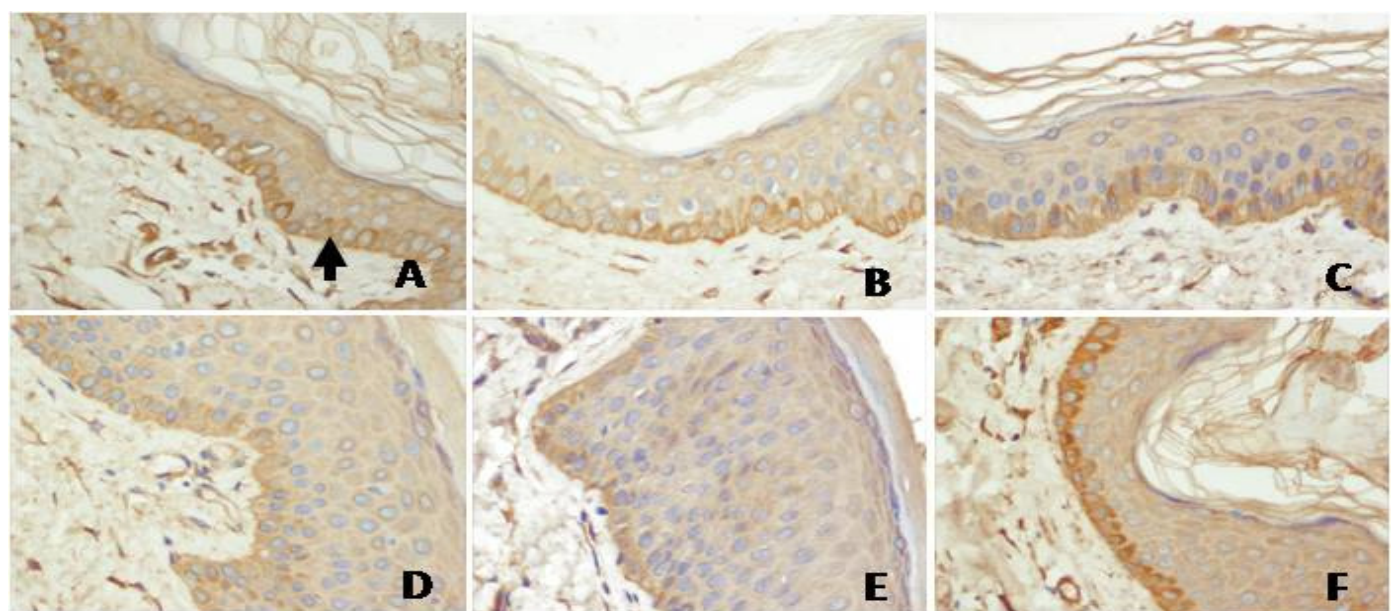

Figure 2. Immunohistochemistry for CK20, CK19 and CK18 in the affected skins of Psoriasis patients $(400 \times) \mathrm{N}$ : normal skin; arrow represents positive staining. A: CK20-N; B: CK19-N; C: CK18-N; D: CK20; E: CK19; F: CD18.

lesioned skin and the region that skin pigment changed after the recovered of lesioned skin was basically to simulate the formation, development and restoration of lesions in psoriatic patients. The neuroendocrine granules in 20 Merkel cells (How many fields?) in each sample from each group were counted and the average was calculated. Results showed the number of granules was $60.21 \pm 12.56$ in the initial stage group, $126.35 \pm 15.57$ in the progressive stage group and $57.39 \pm 14.02$ in the recovery stage group.

Statistical analysis showed the number of granules in the progressive stage group was significantly different from that in the initial and recovery stage groups $(P<0.05)$, but no significant difference was noted between the initial stage group and the recovery stage group $(P>0.05)$.

\section{Expressions of CK18, CK19 and CK20 in the lesioned skins}

Immunohistochemistry indicated that Merkel cells mainly located in the basal cell layers. The expressions of CK18, CK19 and CK20 were found in both the affected skins and the normal skins (Figure 2). Analysis revealed the average optical densities of the lesions positive for CK18, CK19 and CK20 were dramatically higher than those in the normal skins $(P<0.05)$ (Table 1$)$.

Immunohistochemistry for CK20, SI00A7, SP and CD13 in the affected lesions at different stages

The positive particles were brown in the immunohistochemistry and mainly located in the cytoplasm. The positive staining was found in all 3 groups. The optical density of the lesions positive for CK20 was determined. Results indicated that there was significant difference in the expressions of CK20, between the progressive stage group and the remaining two groups $(P<0.05)$, but no marked difference was noted between initial stage group and recovery group $(P>0.05)$. The S100A7 positive particles at initial stage and recovery stage mainly located in the basal layers of the 
Table 1. Expressions of CK20, CK19 and CK18 in the immunohistochemistry (mean optical density).

\begin{tabular}{lccc}
\hline & Normal skin & affected skins & P \\
\hline CK20 & $0.02567 \pm 0.0086$ & $0.0672 \pm 0.0132$ & $<0.05$ \\
CK19 & $0.0221 \pm 0.0071$ & $0.0556 \pm 0.0091$ & $<0.05$ \\
CK18 & $0.0231 \pm 0.0101$ & $0.0701 \pm 0.0211$ & $<0.05$ \\
\hline
\end{tabular}

Table 2. Expressions of CK20, S100A7, SP and CD13 (immunohistochemistry) in the affected skins at different stages of psoriasis (mean optical density).

\begin{tabular}{lcccc}
\hline & n & Initial stage & progressive stage & recovery stage \\
\hline CK20 & 19 & $0.0417 \pm 0.0243$ & $0.0678 \pm 0.0174^{*}$ & $0.0351 \pm 0.0134^{*}$ \\
S100A7 & 19 & $0.0354 \pm 0.0151$ & $0.0832 \pm 0.031^{*}$ & $0.0392 \pm 0.0167^{*}$ \\
SP & 19 & $0.0256 \pm 0.0087$ & $0.0588 \pm 0.0142^{*}$ & $0.0223 \pm 0.0122^{*}$ \\
CD13 & 19 & $0.0242 \pm 0.0084$ & $0.0615 \pm 0.0198^{*}$ & $0.0284 \pm 0.0109^{*}$ \\
\hline
\end{tabular}

${ }^{*} \mathrm{P}<0.05$ vs initial stage group and recovery stage group; ${ }^{*} \mathrm{P}>0.05$ vs initial stage group.

epidermis, but those at progressive stage could be observed in the whole layer of the epidermis. Analysis indicated that there was significant difference in the S100A7 expression between the progressive stage group and the initial or recovery stage group $(P<0.05)$ but no significant difference was noted between the initial stage group and recovery stage group $(P>0.05)$. Our results also indicated that there were significant differences in the expressions of SP and CD13 between the progressive stage group and the initial or recovery stage group $(P<0.05)$ but no significant difference between the initial stage group and recovery stage group $(P>0.05)$ (Figure 3 and Table 2).

\section{Correlation between the CK20 expression and the expressions of SI00A7, SP and CD13}

Correlation analysis showed the CK20 expression was closely related to the expressions of SIO0A7, SP and CD13 $(r=0.7854,0.7201$ and 0.6423 , respectively; $\mathrm{P}<0.05)$.

\section{DISCUSSION}

Merkel cells, firstly described by Merkel in 1875, mainly localize in the basal layer of the epidermis, and are closely connected to the nerve endings with clear cytoplasm and irregular nucleus. These cells are distributed between the epidermal cells with digitations connected by desmosomes. They had the features of epidermal cells and granules of $50 \sim 100 \mathrm{~nm}$. The enlarged nerve endings are wrapped in the cytoplasm of Merkel cells. The enlarged nerve endings that are connected to Merkel cells are myelinated nerve fibers (nerve fiber type $A_{\delta}$ ) while those wrapped by Merkel cells are unmyelinated nerve fibers (nerve fiber type $\mathrm{C}$ ). Both nerve fibers respond to various stimulations including physical stimulations (trauma, heat, coldness, vascular permeability changes, swelling, mechanic stimulations and ultraviolet) and chemical stimulations (toxins, allergens, proteases and microorganisms) (Steinhoff et al., 2003). These detrimental stimulations have been attributed to the pathogenic factors of psoriasis and Merkel cells possess the function to receive and transmit the information from these pathogenic factors of psoriasis. It has been found that Merkel cells are relevant with neuroendocrine. Under an electron microscope, neuroendocrine granules can be found in the cytoplasm of Merkel cells, and these cells can secret a lot of neuropeptides such as NGF, SP, VIP and CGRP.

In addition, as Merkel cells have the receptors of SP, VIP and CGRP (Tachibana and Nawa, 2005), and there are desmosomes connections between Merkel cells and keratinocytes, so the SP, VIP and CGRP may deliver the stimulation information to keratinocytes through the desmosomes connections between Merkel cells and keratinocytes and to stimulate keratinocytes secreting IL1, IL-8 and TNF- $\alpha$ (Dallos et al., 2006). Studies revealed that Merkel cells can not only receive and transmit the information from the pathgenic factors of psoriasis, but also conduct the regulatory signals to the advanced central nervous system by the way of neuroendocrine. Early in 1992, Wollina found that the number of Merkel cells and neuropeptides in lesioned skins of psoriasis patients was different from those in the normal skins (Wollina and Mahrle, 1992). It was accepted that number of Merkel cells in the skin is small and these cells have little effect on the physiological function. Actually, the 


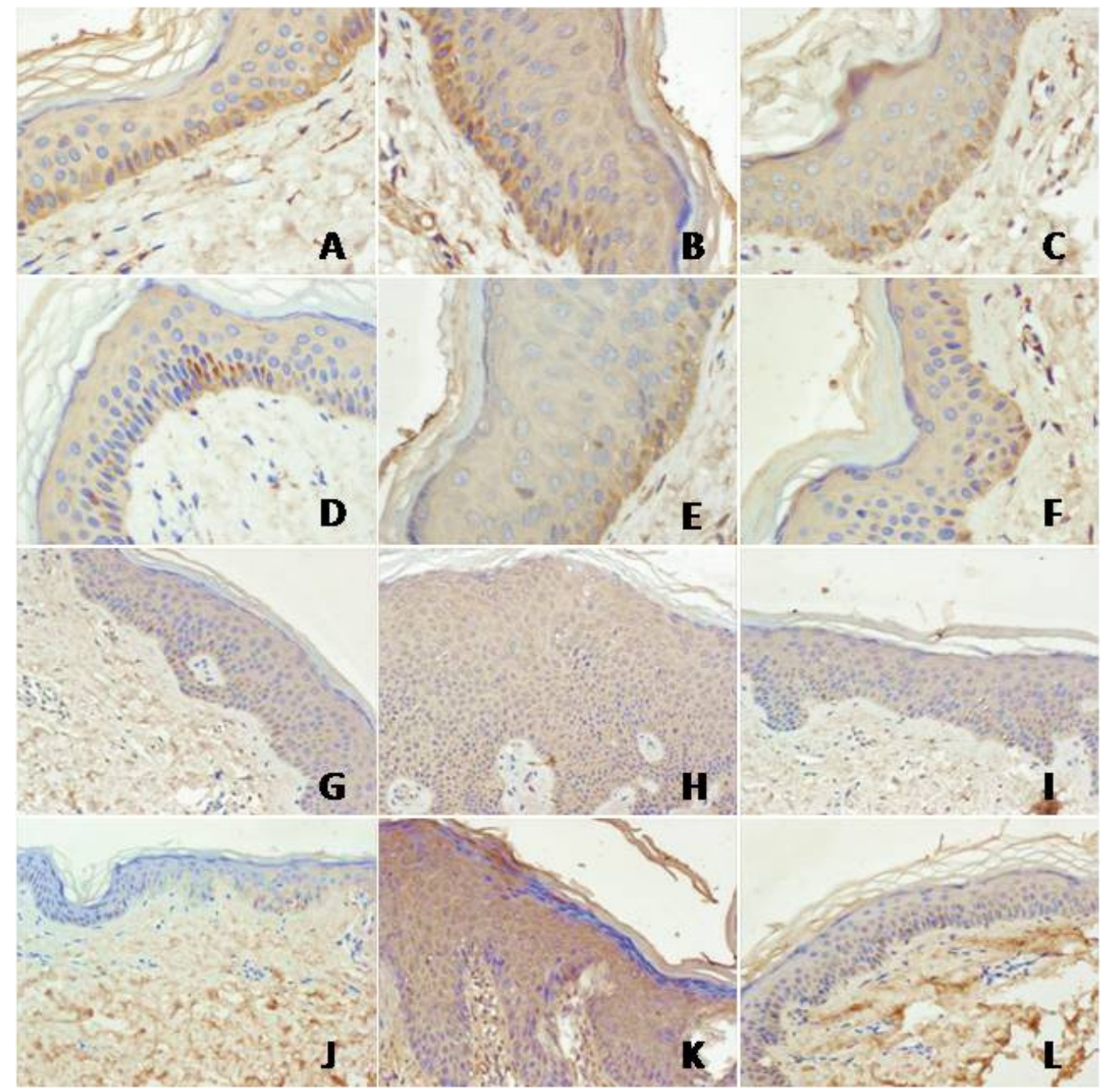

Figure 3. Expressions of CK20, S100A7, SP and CD13 in the affected skins at different stages of psoriasis. A: initial stage-CK20; B: progressive stage-CK20; C: recovery stage- CK20 (400x); D: initial stage-S100A7; E: progressive stage-S100A7; F: recovery stage-S100A7 $(400 \times)^{\prime} \mathrm{G}$ : initial stage-SP; H: progressive stage-SP; I: recovery stage-SP. (200x); J: initial stage-CD13; K: progressive stage-CD13; L: recovery stage-CD13 (200x).

number of Merkel cells in human body is large. Fradette et al. (2003) labeled human Merkel cells with CK20 and results revealed Merkel cells acounted for $3.6 \sim 5.7 \%$ of cells in the basal layer of the epidermis, and there was no difference in the number of Merkel cells between hairy zone or hairless zone. Therefore, the regulatory effect of Merkel cells on human skins should not be neglected.

Our results indicated that the expressions of CK20, CK19 and CK18 in affected skins of psoriasis patients were significantly higher than those in the normal skins, which suggests the Merkel cells in the lesioned skins of psoriasis patients are proliferating. Immunohistochemistry for CK20 revealed a significant difference between the progressive stage group and the initial or recovery stage group. Electron microscopy revealed the number of neuroendocrine granules in the Merkel cells of affected skins of psoriasis patients was dramtically higher than that in the normal skins, and the expressions of S100A7 SP and CD13 in lesions were also markedly increased when compared with those at initial and recovery stage groups. SP is a major neuropeptide and can activate T cells, induce the expression of adhesion molecules and activate endothelial cells and neutrophils to produce interleukin (IL-8). SP can specifically exert effect on the peripheral blood mononuclear cells and promote the synthesis and secretion of IL-6, TNF- $\alpha$ and other cytokines by these cells. IL- 6 and TNF- $\alpha$ can promote the proliferation of KCs in different ways (Milner et al., 2004; Ogata et al., 1999; Pfundt et al., 2000). S100A7 mainly locates in human epidermal KCs is one of participants in the antimicrobial defense system of psoriasis patients, and participate in the regulation of innate immune in the pathogenesis of psoriasis. S100A7 has been shown to function as a chemokine and/or as a cytokine to attract 
$\mathrm{CD}^{+}$lymphocytes and neutrophils, and is mainly present in the cell periphery of terminally differentiated keratinocytes (Broome et al., 2003; Eckert et al., 2004). CD13 is a cell membrane glycoprotein and also called Aminopeptidase $\mathrm{N}$ as it can hydrolyze amino acid from the end $\mathrm{N}$ of peptide, amide and aryl amide. It has been found that $\mathrm{CD} 13$ can regulate the activation of $T$ lymphocytes, the proliferation and secretion of cytokines, and their inhibitors are of potential effect on psoriasis, skin keratosis abnormities and inflammatory diseases (Reinhold et al., 2006; Thielitz et al., 2008).

Our results indicated the expressions of SP, S100A7 and CD13 were positively correlated with the CD20 expression which represents the amount of Merkel cells). This finding suggests that Merkel cells may play a neuroendocrine regulatory role in the pathogenesis of psoriasis. We speculate that Merkel cells may play an important role in the pathogenesis of psoriasis. After receiving endogenous and exogenous signals, on one hand, merkel cells then transmit these signals to advanced CNS through nerve fibers and the advanced CNS then sends out the regulatory signals after analysis and integration. These signals are transmitted back to Merkel cells through nerve fibers or neuroendocrine. On the other hand, Merkel cells secrete neuropeptide activating $T$ lymphocytes through SP and CD13, and induce abnormal proliferation and apoptosis of keratinocytes, as well as abnormities of blood vessels. Merkel cells may also affect the innate immune of psoriasis patients through S100A7. These effects of Merkel cells finally result in the formation of skin damage in psoriasis patients. Our study provides evidence for the concept of neuro-cutaneous-endocrine network in psoriasis patients and beneficial for the in depth studies on the mechanisms of psoriasis.

\section{REFERENCES}

Broome AM, Ryan D, Eckert RL (2003). S100 Protein Subcellular Localization during Epidermal Differentiation and Psoriasis. J. Histochem. Cytochem., 51(5): 675-685.

Dallos A, Kiss M, Polyánka H, Dobozy A, Kemény L, Husz S (2006). Effects of the neuropeptides substance $P$, calcitonin gene-related peptide, vasoactive intestinal polypeptide and galanin on the production of nerve growth factor and inflammatory cytokines in cultured human keratinocytes. Neuropeptides, 40(4): 251-63.

Eckert RL, Broome AM, Ruse M, Robinson N, Ryan D, Lee K (2004). S100 Proteins in the Epidermis. J. Invest. Dermatol., $123: 23-33$.

Fradette J, Larouche $D$, Fugère $C$, Guignard R, Beauparlant $A$, Couture V, Caouette-Laberge L, Roy A, Germain L (2003). Normal human Merkel cells are present in epidermal cell populations isolated and cultured from glabrous and hairy skin sites. J. Invest. Dermatol., 120(2): 313-317.
Karanikas E, Harsoulis F, Giouzepas I, Griveas I (2007). Stimulation of the hypothalamic- pituitary-adrenal axis with corticotropin releasing hormone in patients with psoriasis._HORMONES, 6(4): 314-320.

Karanikas E, Harsoulis F, Giouzepas I, Griveas I, Chrisomallis F (2009). Neuroendocrine stimulatory tests of hypothalamus-pituitary-adrenal axis in psoriasis and correlative implications with psychopathological and immune parameters. J. Dermatol., 36(1): 35-44.

Milner P, Bodin P, Guiducci S, Del Rosso A, Kahaleh MB, MatucciCerinic M (2004). Regulation of substance P mRNA expression in human dermal microvascular endothelial cells. Clin. Exp. Rheumatol., 22(3 Suppl 33): S24-27.

Misery L (1996). Neuro-immuno-cutaneous system (NICS). Pathol. Biol (Paris)., 44(10): 867-874.

Ogata A, Nishimoto N, Yoshizaki K (1999). Advances in interleulcin-6 therapy. Rinsho. Byori., 47(4):321-326.

O'Sullivan RL, Lipper G, Lerner EA (1998). The neuro-immunocutaneous-endocrine network: relationship of mind and skin. Arch. Dermatol., 134(11): 1431-1435.

Pfundt R, Wingens M, Bergers M, Zweers M, Frenken M, Schalkwijk J (2000).TNF-alpha and serum induce KALP/elafin gene expression in human keratinocytes by a p38 MAP kinase-dependent pathway. Arch. Dermatol. Res., 292(4): 180-187.

Reich A, Orda A, Wiśnicka B, Szepietowski JC (2007). Plasma concentration of selected neuropeptides in patients suffering from psoriasis. Exp. Dermatol., 16(5): 421-8.

Reinhold D, Biton A, Pieper S, Lendeckel U, Faust J, Neubert K, Bank U, Täger M, Ansorge S, Brocke S 2006). Dipeptidyl peptidase IV (DP IV, CD26) and aminopeptidase N (APN, CD13) as regulators of T cell function and targets of immunotherapy in CNS inflammation. Int. Immunopharmacol., 6(13-14): 1935-1942.

Roosterman D, Goerge T, Schneider SW, Bunnett NW, Steinhoff M (2006). Neuronal Control of Skin Function:The Skin as a Neuroimmunoendocrine Organ. Physio. Rev., 86(4): 1309-1379.

Shepherd AJ, Downing JE, Miyan JA (2005). Without nerves, immunology remains incomplete - in vivo veritas. Immunology, 116: 145-163.

Steinhoff M, Ständer S, Seeliger S, Ansel JC, Schmelz M, Luger T (2003). Modern aspects of cutaneous neurogenic inflammation. Arch. Dermatol., 139: 1479-1488.

Tachibana T, Nawa T (2005). Immunohistochemical reactions of receptors to met-enkephalin, VIP, substance $P$, and CGRP located on Merkel cells in the rat sinus hair follicle. Arch. Histol. Cytol., 68(5): 383-391.

Thielitz A, Ansorge S, Bank U, Tager M, Wrenger S, Gollnick H, Reinhold D (2008). The ectopeptidases dipeptidyl peptidase IV (DP IV) and aminopeptidase N (APN) and their related enzymes as possible targets in the treatment of skin diseases. Front. Biosci., 13: 2364-2375

Wang Yan ,Li Ping ,Wang Ya-kun (2004). Lesional Skin of Psoriasis Vulgaris number of nerve fibers and their relationship with the Langerhans cells observed. Chin. J. Dermatol., 37(3): 144-146.

Wollina U, Mahrle G (1992). Epidermal Merkel cells in psoriatic lesions: immunohistochemical investigations on neuroendocrine antigen expression. J. Dermatol. Sci., 3(3): 145-150.

Ye P, He R, Wu Z (2005). Psoriasis vulgaris lesions of nerve growth factor receptor expression P75NTR and TrKA. Chin. J. Dermatol., 38(10): 637-639. 